\title{
Reemergence of Chloramphenicol Sensitivity among Salmonella enterica serovars Typhi and Paratyphi : a six year experience in a tertiary care hospital in Nepal.
}

\author{
Rajani Shrestha, Niranjan Nayak, Dharm Raj Bhatta, Deependra Hamal, Supram Hosuru \\ Subramanya, Shishir Gokhale \\ Department of Microbiology, Manipal College of Medical Sciences, Pokhara, Nepal
}

Correspondence E-mail : niruni2000@yahoo.com

\begin{abstract}
Emerging drug resistance among Salmonella Typhi and Paratyphi has become challenging in the treatment of enteric fever. The objective of this study was to determine the antibiotic susceptibility pattern of Salmonella serotypes isolated from patients with enteric fever admitted to Manipal Teaching Hospital, Pokhara,Nepal. A total of 30 Salmonella enterica serovar Typhi, Paratyphi A, and Paratyphi $B$ isolated from cases of typhoid and paratyphoid fever admitted to Manipal Teaching Hospital over a period from January 2012 to March 2018 were investigated. All strains were identified by standard microbiological methods and tested for in vitro antibiotic susceptibility testing,using Kirby-Bauer disc diffusion method following the criteria designed by the Clinical and Laboratory Standards Institute (CLSI 2013). S Typhi was the most predominant amongst all the isolates (18 of 30 i.e. $60 \%$ ), followed by S Paratyphi A (33.3\%, 10 out of 30 ) and S Paratyphi B (6.6\%; 2 out of 30$)$. Overall, $91.3 \%$ of the isolates were susceptible to chloramphenicol. The percentage sensitivities towards ceftriaxone, ciprofloxacin, cotrimoxazole and ampicillin were $82.6 \%, 75.8 \%, 63 \%$ and $37 \%$ respectively. All the isolates were sensitive to imipenem and amikacin. These findings suggested that there were changing patterns of antibiotic resistance in enteric fever with reemergence of chloramphenicol sensitive Salmonellae. This necessitates continuous surveillance of cases and re-evaluation of chloramphenicol therapy in Salmonella infections in Nepal.
\end{abstract}

Keywords: Enteric fever; Salmonella; Chloramphenicol; Re-emergence of chloramphenicol sensitivity.

\section{Introduction}

Enteric fever caused by Salmonella enterica serotypes Typhi and Paratyphi is a growing concern worldwide. (Parry, 2010) World Health Organization (WHO) estimated more than 33 million cases of enteric fever globally every year. (Crump et al., 2004) Enteric fever is still one of the serious public health problems in many geographic areas and is endemic in most developing countries. (Prashant, 2010)
This disease is endemic in Asia, Africa, Latin America, the Caribbean islands, and Oceania; majority of cases being reported from Bangladesh, China, India, Indonesia, Laos, Nepal, Pakistan, or Vietnam. (Chau et al., 2007)

In Nepal, enteric fever has popularly been known, since ancient times, in the name of "bisham joauro" meaning fever with poison. In 
Nepal, this disease is prevalent in mountains, valleys and southern belts as an endemic disease with its peak incidence during May to August and is one of the leading causes of fever of unknown origin encountered in most of the hospitals. (Rauniar et al., 2000; Sharma et al., 2003)

Chloramphenicol had been the "gold standard" of therapy for enteric fever since its introduction in 1948. However, Salmonella enterica serovars Typhi and Paratyphi A acquired resistance to chloramphenicol and other antimicrobial agents during the period from 1978-1990 in many countries, causing a major setback in the management of enteric fever cases. (Parry et al., 2002) Thus, ciprofloxacin continues to be the mainstay in the treatment of enteric fever in many places as it is orally effective and economical. (Ishaleku et al., 2015) With increasing use of ciprofloxacin instead of chloramphenicol, resistance to ciprofloxacin showed an upsurge. (Mandal et al., 2004)

Thus, there is a growing concern over enteric fever treatment in Nepal because of rapid development of fluoroquinolone resistance among Salmonella isolates. (Karki et al., 2013; Khanal et al., 2007)

However, discontinuation of chloramphenicol therapy relieved the selection pressure paving the way for re-emergence of Salmonella strains sensitive to chloramphenicol. From 2006 onwards, resistance of Salmonella serotypes to chloramphenicol showed a decline, which was attributable to non-usage of the drug for a long duration of time (Arora et al., 2010) or due to emergence of de novo susceptible strains.

The trend of multidrug resistance Salmonellae showing susceptibility towards conventional first line drugs provided an opportunity to reevaluate the first line traditional drugs as possible therapeutic alternatives.

Thus, the aim of this retrospective study was to analyze drug sensitivity pattern of blood culture isolates in cases of enteric fever at Manipal Teaching Hospital over a period of more than 6 years and to look for any reemergence of sensitivity towards chloramphenicol.

\section{Materials and Methods}

This was a retrospective study carried out at the Manipal Teaching Hospital, Pokhara over a period of more than six years (January 2012 - March 2018). A total of 30 Salmonella enterica serovar Typhi, Paratyphi A and Paratyphi $B$ isolates from as many blood culture positive cases of enteric fever were analyzed.

All strains were identified by conventional biochemical tests and confirmed by serotyping (Khanal et al., 1994) using Salmonella agglutinating sera (Seiken Laboratories, Tokyo, Japan). Antibiotic sensitivity testing was carried out by the standard Kirby Bauer disc diffusion method, (Bauer et al., 1966) using the following antibiotics (conc. /disc): chloramphenicol $(30 \mu \mathrm{g})$, ampicillin $(10 \mu \mathrm{g})$, ciprofloxacin $(5 \mu \mathrm{g})$, ceftriaxone $(30 \mu \mathrm{g})$, cotrimoxazole $(25 \mu \mathrm{g})$, imipenem $(10 \mu \mathrm{g})$ and amikacin $(30 \mu \mathrm{g})$.

\section{Results}

Thirty isolates were obtained over the period from Jan 2012 to March 2018. Salmonella enterica serovar Typhi was isolated from 18 patients, Salmonella enterica serovar Paratyphi A from 10 patients and Paratyphi B from 2 patients; Salmonella enterica serovar Typhi being the most common, accounting for $60 \%(18 / 30)$ of all the isolates.

Figure 1. Antibiotic sensitivity pattern of the isolates

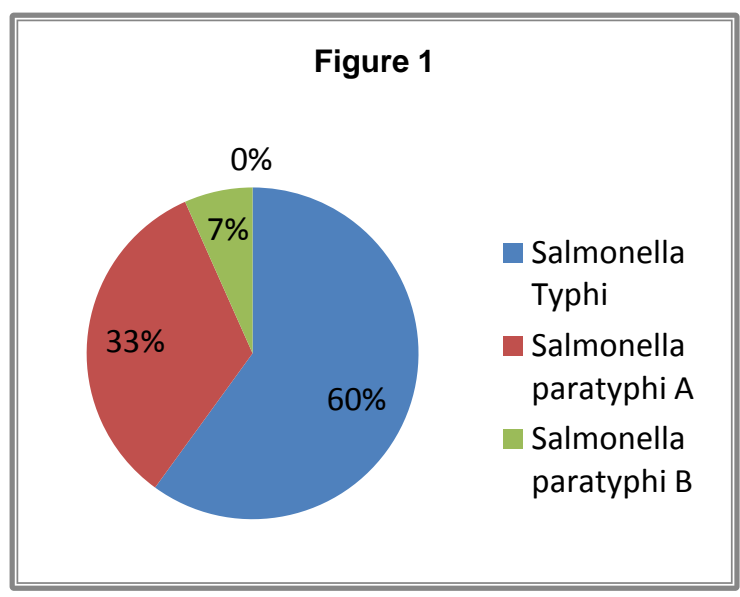

All patients were in the age group of 2-59 years, commonest age group being 10-25 years. Sensitivity pattern of the isolates has been depicted vide figure 2 . 
Figure 2. Percentage isolates of sensitive and resistant Salmonella serotypes

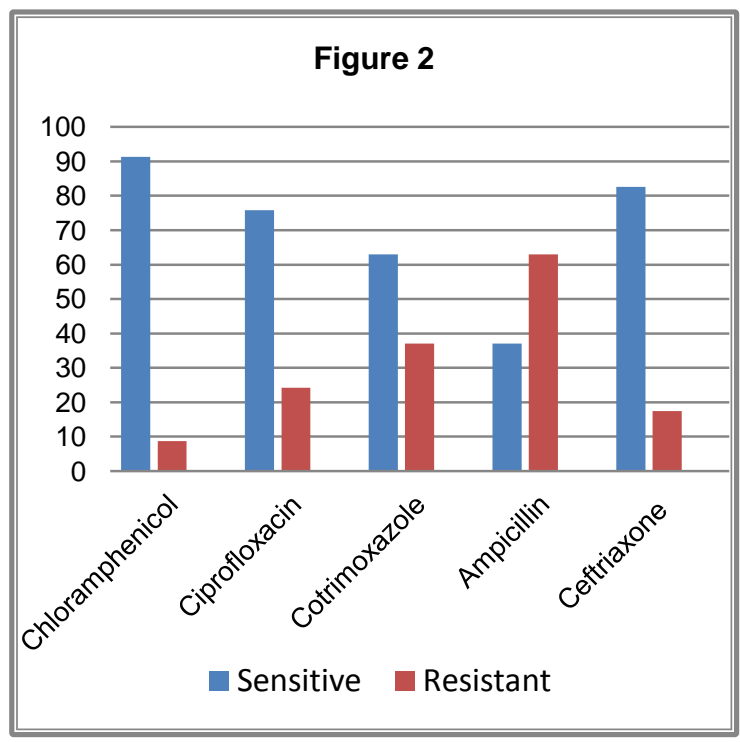

It was interesting to note that $91.3 \%$ of the isolates were susceptible to chloramphenicol. The percentage sensitivities to ceftriaxone, ciprofloxacin, cotrimoxazole and ampicillin were $82.6 \%, 75.8 \%, 63 \%$ and $37 \%$ respectively. All the isolates were sensitive to imipenem and amikacin.

\section{Discussion:}

Enteric fever is a major public health problem in many developing countries. This disease remains as one of the commonest cause of fever in most parts of the developing world including Nepal. (Parry et al., 2010; Maskey et al., 2006).

Chloramphenicol was the mainstay of treatment for enteric fever until 1989 following which MDR strains (resistant to ampicillin, chloramphenicol, cotrimoxazole) were reported worldwide in the year 1990 with the emergence of plasmid R type ACCoSuTTm H1 incompatibility group. (Capoor et al., 2010; Pokharel, et al., 2006) Since then ciprofloxacin was introduced as the first line therapy providing good alternative for the multidrug resistant (MDR) enteric fever pathogens. (Daga, et al., 1994) Thereafter, there were several encouraging reports on the therapeutic efficacy of ciprofloxacin with the clinical evidence of subsidence of fever after a mean of 3 days ciprofloxacin treatment. (Sood et al., 1999).
Subsequently, nalidixic acid resistant Salmonella typhi with decreased susceptibility to ciprofloxacin emerged causing therapeutic failure worldwide and it became endemic in many parts of world with the consequence that high level ciprofloxacin resistance enteric fever evolved in many Asian countries. (Capoor et al., 2010) This was exemplified by the observations of cases in whom fever subsided only after 5-6 days of ciprofloxacin therapy. (Sood et al., 1999) In addition community based studies conducted in India reported that $21.9 \%$ of cases took longer than 8 days to become afebrile and 9 percent cases did not respond to ciprofloxacin even after 15 days of therapy despite in vitro sensitivity. (Crump et al., 2004) Over and above, studies conducted earlier in India and Nepal documented that first and second generation quinolones had varying results over the in vitro activity against $S$. typhi and $S$ paratyphi. (Pokharel et al., 2006; Joshi $S$ et al., 2007)

In context to the above, we looked for the overall sensitivity of our isolates towards both newer and older generation of antityphoidal agents. It was observed that, percentage sensitivity towards chloramphenicol amongst the isolates was as high as $91.3 \%$. This observation was similar to those reported earlier by Prajapati et al from Nepal. (Prajapati et al., 2010) In yet another study in Nepal, Bhatia and colleagues found that 100 percent of Salmonella enterica serovar Paratyphi A and $96 \%$ of Salmonella enterica serovar Typhi were sensitive to chloramphenicol (Bhatia et al., 2007). In a study from Nigeria, Ishaleku et al observed that $85.9 \%$ of their S. typhi and $86.4 \%$ S. paratyphi were susceptible to chloramphenicol (Ishaleku et al., 2015).

Such reemergence of chloramphenicol sensitivity in the recent years, could be due to the omission of chloramphenicol from the treatment regime of enteric fever, which could have led to the withdrawal of selective pressure. Thus resistant bacteria no longer had the advantage of survival in such settings. Reemergence of chloramphenicol sensitive Salmonellae may suggest the necessity for continuous surveillance of enteric fever cases in different geographical areas across the country, as well as globally, to keep abreast 
with the emerging pattern of drug sensitivity among enteric fever pathogens.

Considering the changing drug sensitivity pattern (sensitive to resistance and vice-versa) it is advisable to have continued surveillance of resistance pattern of isolates which would help deciding rational use of antibiotics in the management of enteric fever in near future. In view of the reemergence of chloramphenicol sensitivity among $S$ typhi and $S$ paratyphi isolates in many parts of the world, this drug may be reconsidered as the antibiotic of choice in the treatment of enteric fever.

\section{Conclusion}

Our findings suggested reemergence of chloramphenicol sensitivity among the blood culture isolates of Salmonellae in Nepal. This highlights the necessity for continuous monitoring of cases and rolling back to chloramphenicol as the antibiotic of choice in the treatment of enteric fever.

\section{References}

Arora, D., Singh, R., Kaur, M., Ahi, R.S. (2010). A changing pattern in antimicrobial susceptibility of Salmonella enterica serotype isolated in north India. African Journal of Microbiology Research.4, 197-203

Bauer, A.W., Kirby, W.M.M., Sherris, J.C.,Turck, M. (1966) Antibotic susceptibility testing by standardized single disc method. American Journal of Clinical Pathology. 45,493-6.

Bhatia, J.K., Mathur, A.D., Arora, M. M (2007) Re-emergence of chloramphenicol sensitivity in enteric fever. Medical Journal Armed Forces India. 63(3), 212-4.

Capoor, M.R. \& Nair, D. (2010) Quinolone and cephalosporin resistance in enteric fever .Journal of Global Infectious disease.2 (3), 258-62.

Chau, T.T., Campbell, J.I., Galindo, C.M., Van Minh Hoang, N., Diep, T.S., Nga, T.T. et al. (2007) Antimicrobial drug resistance of Salmonella enterica serovar Typhi in Asia and molecular mechanism of reduced susceptibility to the fluoroquinolones. Antimicrobial Agents and Chemotherapy. 51(12), 4315-23.
Crump, J.A., Luby, S.P., Mintz, E.D. (2004). The global burden of typhoid fever. Bulletin World Health Organization. 82(5), 346-53.

Daga, M.K., Sarin, K., Sarkar, R. (1994). A study of culture positive multidrug resistant enteric fever-changing pattern and emerging resistance to ciprofloxacin. Journal of the Association of Physicians of India. 42(8), 599600.

Ishaleku, D., Umeh, E.U., Amali, O., Gberikon, G.M. (2015). The re-emergence of chloramphenicol sensitive Salmonella species among typhoid fever patients in the southern geographical zone of Nasarawa state, Nigeria. Journal of Infectious Disease and Therapy. $3(3), 586-7$

Joshi, S. \& Amarnath, S.K. (2007) Fluoroquinolone resistance in Salmonella Typhi and $S$ Paratyphi A in Bangalore, India. Transactions of the Royal Society of Tropical Medicine and Hygiene. 101(3), 308-10.

Khanal, B., Sharma, S. K., Bhattacharya, S.K., Bhattarai, N.R., Deb, M.,Kanungo,R. (2007). Antimicrobial susceptibility patterns of Salmonella enterica serotype Typhi in eastern Nepal. Journal of Health, Populaton and Nutriton. 25(1), 82-7.

Karki, S., Shakya. P., Cheng, A.C., Dumre, S.P., Leder, K. (2013). Trends of etiology and drug resistance in enteric fever in the last two decades in Nepal: A systematic review and metaanalysis. Cinical Infectious Disease. 57 (10), e167-76.

Mandal, S., Mandal, M.D., Pal, N.K. (2004) Reduced minimum inhibitory concentration of chloramphenicol for salmonella enterica serovar typhi. Indian Journal of Medical Sciences. 58 (1), 16-23.

Maskey A.P., Day, J.N., Phung,Q.T., Thwaites, G.E., Campbell, J.I., Zimmerman, M. et al. (2006) Salmonella enterica serovar Paratyphi $A$ and $S$. enteric serovar Typhi cause indistinguishable clinical syndromes in Kathmandu, Nepal. Clinical Infectious Disease. 42(9), 1247-53.

Parry, C.M., Thuy, C.T., Dongol, S., Karkey, A., Vinh, H., Chinh, N.T. et al. (2010). Suitable disk antimicrobial susceptibility breakpoints defining Salmonella enterica serovar Typhi 
isolates with reduced susceptibility to fluoroquinolones. Antimicrobial Agents and Chemotherapy. 54(12), 5201-8.

Parry, C. M., Hien, T.T., Dougan, G., White, N.J., Farrar, J.J. et al. (2002) Typhoid Fever. New England Journal of Medicine. 347(22), 1770-82.

Prashant Khandeparkar. (2010) Reemergence of Chloramphenicol in Typhoid Fever In the Era of Antibiotic Resistance.SUPPLEMENT TO JAPI. (58), 45-46.

Prajapati, B., Rai, G. K., Rai, S. K., Upreti, H.C., Thapa, M., Singh, G., Shrestha, R. M. (2008) Prevalence of Salmonella typhi and paratyphi infection in children: a hospital based study; Nepal Medical College Journal.10 (4): 238-41.

Pokharel, B.M., Koirala, J., Dahal, R.K., Mishra, S.K., Khadga, P.K., Tuladhar, N.R. et al. (2006) Multidrug resistant and extended spectrum beta lactamase (ESBL) producing Salmonella enterica (serotypes Typhi and paratyphi A) from blood isolates in Nepal: Surveillance of resistance and a search for newer alternatives. International Journal of Infectious Disease. 10(6), 434-8.
Rauniar, G.P., Das, B.P., Baral, D.D., Naga Rani, M.A. et al. (2000) Treatment pattern of typhoid fever at a tertiary care teaching hospital in eastern Nepal. Journal of Nepal Medical Association. 39, 218- 21.

Sharma, N., Koju, R., Karmacharya, B., Tamang, M. D., Makaju, R., Nepali, N., et al. (2003). Typhoid fever in Dhulikhel hospital, Nepal. Kathmandu University Medical Journal. 2(3), 188-92

Sood, S., Kapil, A., Das, B., Jian, Y., Kabra, S. K. (1999) Reemergence of chloramphenicol sensitive Salmonella typhi. Lancet. 353(916), 1241-2. 